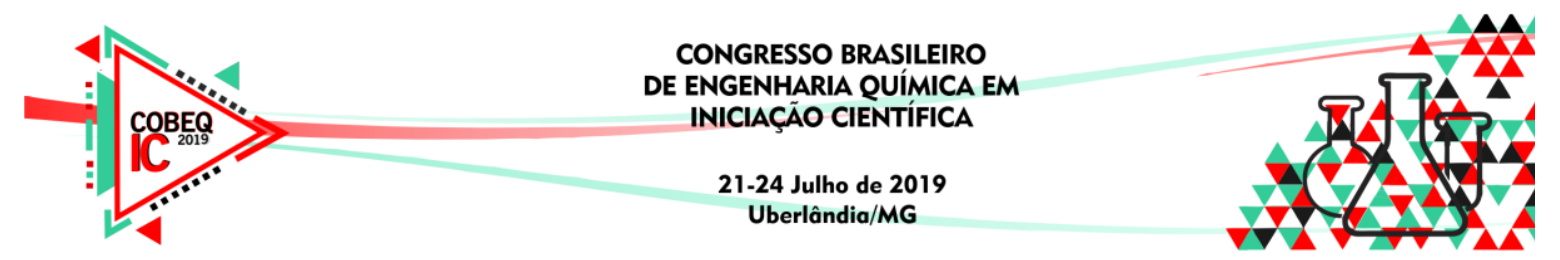

\title{
ESTUDO DE ANCORAMENTO MOLECULAR (DOCKING) DE POTENCIAIS INIBIDORES DA BUTIRILCOLINESTERASE HUMANA (HssBChE)
}

\author{
E. D. S. FERRO ${ }^{1}$, A. P. GUIMARÃES ${ }^{2}$ e A. S. L. GONÇALVES ${ }^{3}$ \\ ${ }^{1}$ Faculdade de Ciências e Tecnologia de Viçosa, Departamento de Engenharia Química \\ 2Universidade Federal de Viçosa, Departamento de Química \\ ${ }^{3}$ Universidade Federal de Minas Gerais, Departamento de Química \\ E-mail para contato: ellen_rezendeferro@outlook.com
}

\begin{abstract}
RESUMO - A butirilcolinesterase (BChE) é uma enzima presente no sistema nervoso que está diretamente relacionada a doença de Alzheimer (DA). Essa enfermidade está associada à perda seletiva de neurônios colinérgicos no cérebro e a níveis reduzidos do neurotransmissor acetilcolina $(\mathrm{ACh})$, provocando senilidade precoce. Atualmente, o tratamento da DA baseia-se na inibição da acetilcolinesterase humana (Hss AChE) por compostos que reduzem significativamente os sintomas da doença. Todavia, a BChE também está sendo vista como um alvo potencial devido a seu papel na modulação dos níveis de ACh nos neurônios colinérgicos. Infelizmente a DA ainda não tem cura, sendo assim, frente ao gigantesco potencial danoso associado a essa doença, torna-se extremamente necessário o desenvolvimento de pesquisas pela descoberta de novos fármacos que sejam capazes de combatê-la, uma vez que, os fármacos existentes provocam diversos efeitos colaterais. Neste contexto, no presente trabalho, realizamos um estudo por docagem molecular (docking) entre a $H s s \mathrm{BChE}$ e 8 compostos propostos por (CHEN, et al. 2018), com o objetivo de investigar suas interações e consequente possível poder inibitório da $H s s \mathrm{BChE}$. A metodologia foi validada através do re-docking da tacrina na HssBChE. Dentre os compostos estudados o Corylifol A e o Neobavaisoflavone apresentaram maior estabilidade no sitio ativo da $H s s \mathrm{BChE}$.
\end{abstract}

\section{INTRODUÇÃO}

A butirilcolinesterase (BChE) juntamente com a acetilcolinesterase (AChE) são as enzimas colinesterásicas encontradas no sistema nervoso que juntas catalisam a hidrólise da acetilcolina (ACh), diminuindo os níveis do neurotransmissor e finalizando a comunicação entre as células nervosas; resultando na diminuição da função cerebral e consequentemente a perda de habilidades intelectuais. A BChE tem menor afinidade pela ACh se comparado a $\mathrm{AChE}$, porém é crucial para o processo quando há presença de altos níveis de $\mathrm{ACh}$ (ZILBEYAZ, 2018).

A diminuição dos neurotransmissores está diretamente relacionada a doença de Alzheimer (DA), sendo a causa mais comum de demência e responsável por cerca de $60-80 \%$ de todas as demências. A DA leva os pacientes a estresse emocional, distúrbios comportamentais e grande sofrimento (BONO, 2014). 


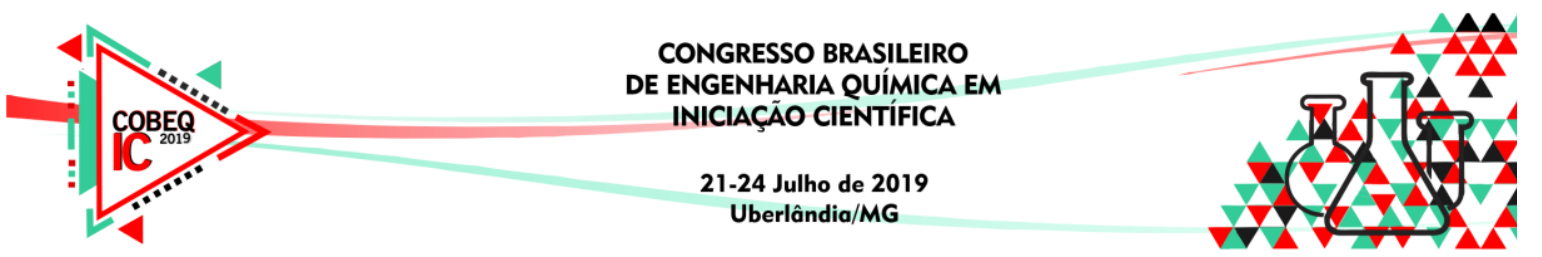

Atualmente, o tratamento da DA baseia-se na inibição da AChE por compostos que reduzem significativamente os sintomas da doença. Drogas sintomáticas como tacrina, donepezil, galantamina e rivastigmina são utilizadas para o tratamento, uma vez que inibem a atividade da $\mathrm{AChE}$ e consequentemente aumenta o nível da $\mathrm{ACh}$. A figura 1 apresenta as estruturas dos fármacos aprovados para o tratamento da DA.

Figura 1 - Estruturas dos fármacos aprovados para tratamento da DA.

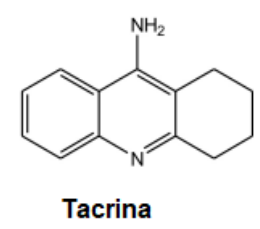

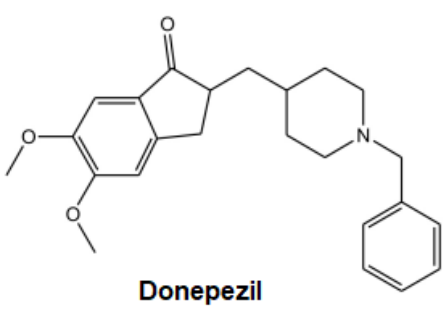

Donepezil

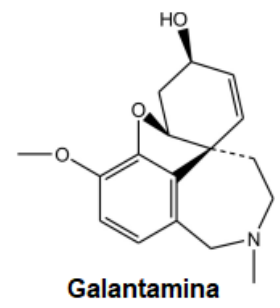

Galantamina<smiles>CCN(C)C(=O)Oc1cccc([C@H](C)N(C)C)c1</smiles>

Rivastigmina

A tacrina foi o primeiro fármaco usado em larga escala para o tratamento da DA, sendo um inibidor reversível e de abordagem terapêutica eficaz, porém a administração do fármaco necessita de quatro doses diárias o que possibilita o aumento das enzimas hepáticas em torno de cinco vezes o valor normal (VALE et al, 2011). Outro fármaco amplamente utilizado é o donepezil, assim como a tacrina, é um inibidor reversível, cuja dose é diária e vem se mostrando mais potente que a tacrina em estudos in vitro. Este fármaco inibi seletivamente a $\mathrm{AChE} \mathrm{em}$ vez da BChE, ocasionando o aumento nos níveis de ACh (HOWARD, R et al, 2012).

A galantamina é um alcaloide inibidor competitivo de curta duração que se liga reversivelmente à $\mathrm{AChE}$, e atua como modulador dos receptores nicotínicos, potencializando ainda mais a neurotransmissão colinérgica (BONO, 2014). Contudo, o fármaco atualmente mais utilizado para o tratamento da DA é a rivastigmina, capaz de inibir tanto a AChE quanto a $\mathrm{BChE}$, apresentando maior eficácia no aumento dos níveis de ACh no cérebro. No entanto, ambos os medicamentos (donepezil e rivastigmina) ocasionam vários efeitos colaterais, como náusea, vômito, diarreia, e dor abdominal o que ocasiona o abandono da medicação pelos pacientes (INOUYE, 2003).

Mediante o exposto, neste trabalho utilizamos a $H s s \mathrm{BChE}$ como um possível alvo para realizamos estudos por docking para prever o poder inibitório de 8 potenciais inibidores propostos por Chen e colaboradores (CHEN, et al. 2018). Após os estudos foi possível identificar os compostos mais promissores. Com base nos resultados obtidos iremos propor modificações estruturais nos compostos estudados, objetivando contribuir de forma significativa para o planejamento de novos protótipos mais eficazes e seletivos para $H s s \mathrm{BChE}$ e consequentemente novos fármacos contra DA.

\section{METODOLOGIA}

No presente trabalho, o processo de reconhecimento molecular entre a HssBChE (PDB: 4BDS) e 8 potenciais inibidores propostos por Chen e colaboradores (CHEN, et al. 2018) foi estudado utilizando-se docking. A figura 2 apresenta as estruturas dos compostos estudados. 




Figura 2 - Estruturas dos compostos estudados (CHEN, et al. 2018).


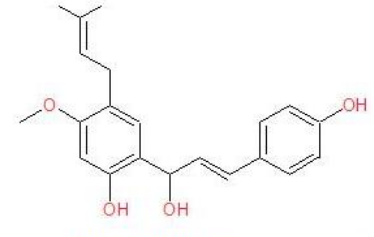

Bavachalvone

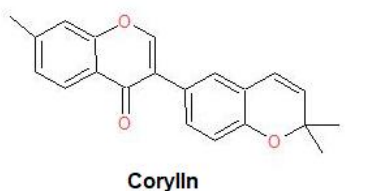

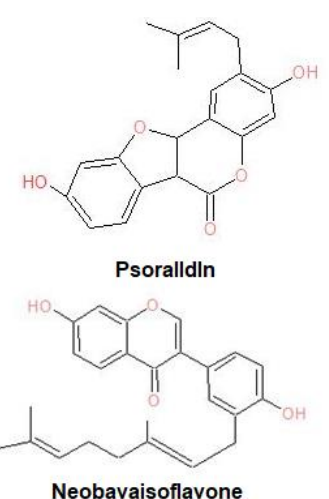

Neobavaisoflavone

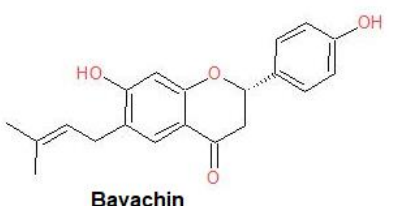

Bavachin

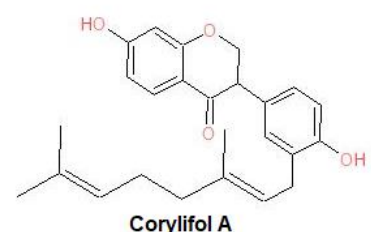

As estruturas tridimensionais dos compostos foram construídas e otimizadas no programa PC Spartan Pro (HEHRE et al., 1999) através de cálculos de mecânica molecular, usando o campo de força Merck Molecular Force Field (MMFF). Sendo em seguida, determinadas as cargas parciais dos átomos, utilizando o método semi-empírico AM1. Os cálculos de docking dos compostos no sítio ativo da $H s s \mathrm{BChE}$ foram realizadas no programa Molegro Virtual Docker (MVD) (THOMSEN E CHRISTENSEN, 2006).

Utilizou-se uma metodologia de docagem flexível, onde permitiu-se a flexibilidade do ligante e da proteína para permitir um melhor reconhecimento molecular, evitando-se alterações durante a formação do complexo ligante-proteína. A metodologia foi validada através do redocking dos 2 compostos já localizados no sitio ativo da enzima, sendo eles tacrina e donepezil.

\section{RESULTADOS E DISCUSSÕES}

Os cálculos de docking foram realizados com o intuito de obter as melhores poses dos compostos estudados no sítio ativo da $H s s \mathrm{BChE}$, a fim de obter informações sobre as interações entre os compostos estudados e a enzima.

\subsection{Validação da metodologia de docking}

Com o intuito de validar a metodologia de docking realizou-se cálculos de re-docking, nos quais as estruturas presentes na enzima (ligantes obtidos da estrutura cristalográfica) são ancoradas sobre si mesma. Primeiramente, realizou-se o re-docking do donepezil na Hss AChE a fim de obter um parâmetro para comparar com o resultado obtido na $H s s \mathrm{BChE}$. O valor de DRMQ (Desvio da raiz média quadrática) obtido do re-docking do donepezil no sítio ativo da $H$ ss AChE foi de 0,301 A. Posteriormente, realizou-se o re-docking da tacrina no sítio ativo da $H s s \mathrm{BChE}$, obtendo um valor de DRMQ igual a 0,156 $\AA$. As poses selecionadas do re-docking tanto da $H s s \mathrm{AChE}$ quanto da $H s s \mathrm{BChE}$ estão apresentadas na figura 3. 


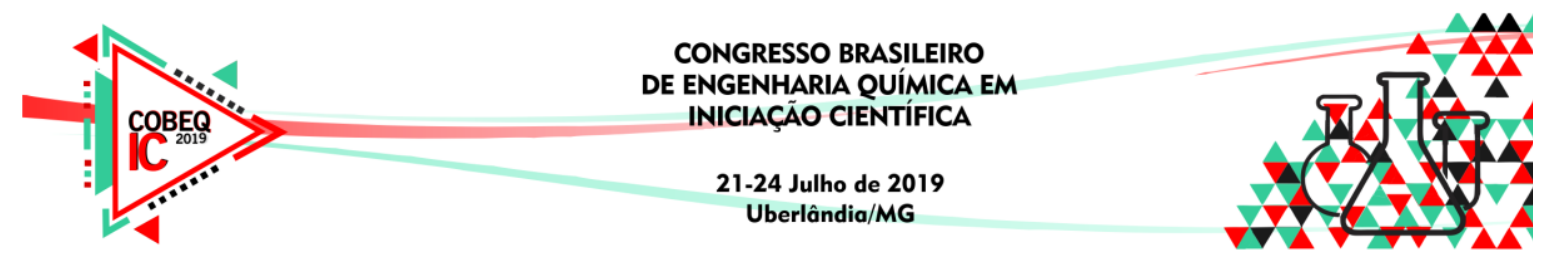

Figura 3 - Poses escolhidas dos cálculos de re-docking do donepezil de amarelo (à esquerda) e da tacrina de amarelo (à direita) em sobreposição as conformaçõos cristalográficas nos sítios de ligação da $H s s \mathrm{AChE}$ e da $H s s \mathrm{BChE}$ respectivamente.
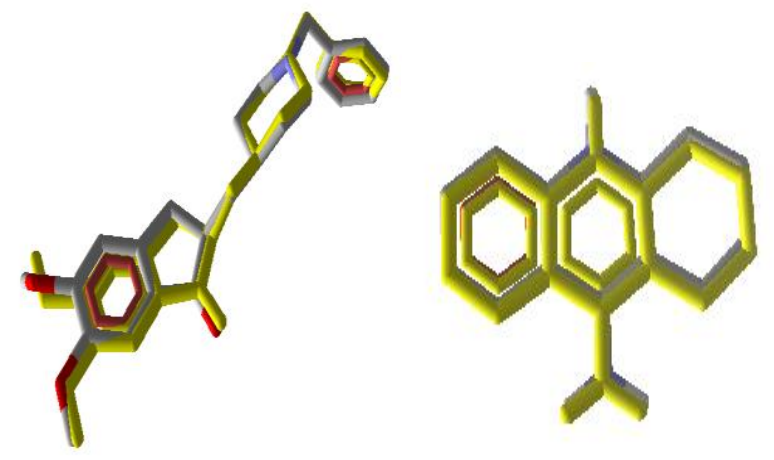

Os baixos valores de DMRQ encontrados juntamente com as configurações obtidas foram satisfatoriamente próximas as estruturas cristalográficas. Estes valores validam a metodologia de docking considerando que, segundo a literatura, um DMRQ menor que 2,0 Å é considerado aceitável, validando assim o protocolo de docking utilizado (KONTOYIANNI et al. 2004).

O donepezil interagiu com o resíduo Phe295 pertencente ao sítio ativo da Hss AChE e com três moléculas de água, já a tacrina interagiu com o His438 e com duas moléculas de água. Os valores obtidos através do re-docking para as energias total de interação intermolecular (EII)

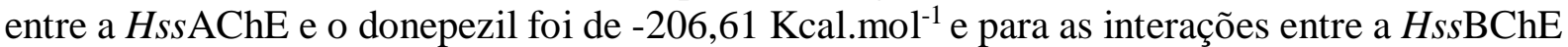
e a tacrina foi de $-103,86 \mathrm{Kcal}_{\mathrm{mol}}{ }^{-1}$.

\subsection{Docking dos compostos estudados}

Os compostos propostos por Chen e colaboradores (CHEN, et al. 2018), foram preliminarmente sugeridos como inibidores da Hss AChE, porém após estudos sobre o papel da Hss BChE no processo de hidrólise da $\mathrm{ACh}$, houve a necessidade de realizar o docking dos compostos na $H s s \mathrm{BChE}$ afim de ampliar os conhecimentos acerca do sítio ativo desta enzima e a capacidade de inibição dos compostos propostos.

Os resultados obtidos do re-docking do donepezil na Hss AChE e da tacrina na HssBChE, juntamente com o docking dos demais compostos estudados são apresentados na tabela 1 . Os resultados apresentados para a $H s s \mathrm{AChE}$ foram obtidos de um trabalho realizado anteriormente (FERRO; GUIMARÃES; GONÇALVES. 2018). 



Tabela 1 - Resultado do docking dos compostos estudados na Hss AChE e na HssBChE.

\begin{tabular}{|c|c|c|c|c|}
\cline { 2 - 5 } \multicolumn{1}{c|}{} & \multicolumn{2}{c|}{ HsAChE } & \multicolumn{2}{c|}{ HssBChE } \\
\hline Composto & $\begin{array}{c}\text { Energia de } \\
\text { Interação } \\
\left(\text { Kcal.mol }^{-1}\right)\end{array}$ & $\begin{array}{c}\text { Energia de Ligação } \\
\text { Hidrogênio } \\
\left(\text { Kcal.mol }^{-1}\right)\end{array}$ & $\begin{array}{c}\text { Energia de } \\
\text { Interação } \\
\left(\text { Kcal.mol }^{-1}\right)\end{array}$ & $\begin{array}{c}\text { Energia de } \\
\text { Ligação } \\
\text { Hidrogênio } \\
\left(\text { Kcal.mol }^{-1}\right)\end{array}$ \\
\hline Donepezil & $-206,61$ & $-2,35$ & $-132,50$ & 0 \\
\hline Tacrina & $-104,53$ & $-2,07$ & $-103,86$ & 2,50 \\
\hline Rivastigmina & $-118,86$ & $-4,46$ & $-94,62$ & $-1,55$ \\
\hline Isobavachalcone & $-181,17$ & $-7,18$ & $-144,66$ & $-8,02$ \\
\hline Bavachalcone & $-197,53$ & $-9,14$ & $-163,06$ & $-7,94$ \\
\hline Psoralldin & $-175,64$ & $-7,00$ & $-150,37$ & $-11,5$ \\
\hline Bavachin & $-175,97$ & $-5,78$ & $-135,47$ & $-7,76$ \\
\hline Bavachinin & $-167,85$ & $-5,39$ & $-139,33$ & $-7,50$ \\
\hline Corylin & $-154,74$ & $-4,49$ & $-128,10$ & 0 \\
\hline Neobavaisoflavone & $-205,49$ & $-5,00$ & $-182,48$ & $-5,95$ \\
\hline Corylifol A & $-207,29$ & $-4,63$ & $-181,72$ & $-5,95$ \\
\hline
\end{tabular}

Com base na tabela acima é possível observar que os compostos estudados apresentaram maior potencial de inibição para a HssAChE. O donepezil ao ser re-docado na Hss AChE apresentou uma energia de interação de $-206,61 \mathrm{Kcal}^{\mathrm{mol}}{ }^{-1}$, valor este muito inferior ao determinado quando o mesmo foi docado na $H s s \mathrm{BChE}\left(-132,5 \mathrm{Kcal}^{\mathrm{mol}}{ }^{-1}\right)$. Portanto, concluímos que a energia necessária para que ocorra a interação do donepezil no sítio ativo é muito maior na HssBChE do que na HssAChE. Resultado análogo é observado para a rivastigmina, contudo a tacrina apresentou energia de interação intermolecular semelhante para ambas as enzimas, tal fato se dá, devido a capacidade deste fármaco de bloquear a hidrólise da ACh (HOWARD, R et al, 2012).

Os compostos estudados apresentaram melhor energia de interação para a Hss AChE em detrimento à HssBChE. Dentre os compostos estudados o Neobavaisoflavone e o Corylifol A apresentaram maior estabilidade ao sítio ativo de ambas as enzimas, o que remete ao elevado potencial de inibição desses compostos. As figuras 4 e 5 apresentam as poses escolhidas para ambos os compostos na HssBChE.

Figura 4 - Pose escolhida para o do Neobavaisoflavone na HssBChE em sobreposição ao donepezil de amarelo (à esquerda) e interações com os resíduos (à direita).
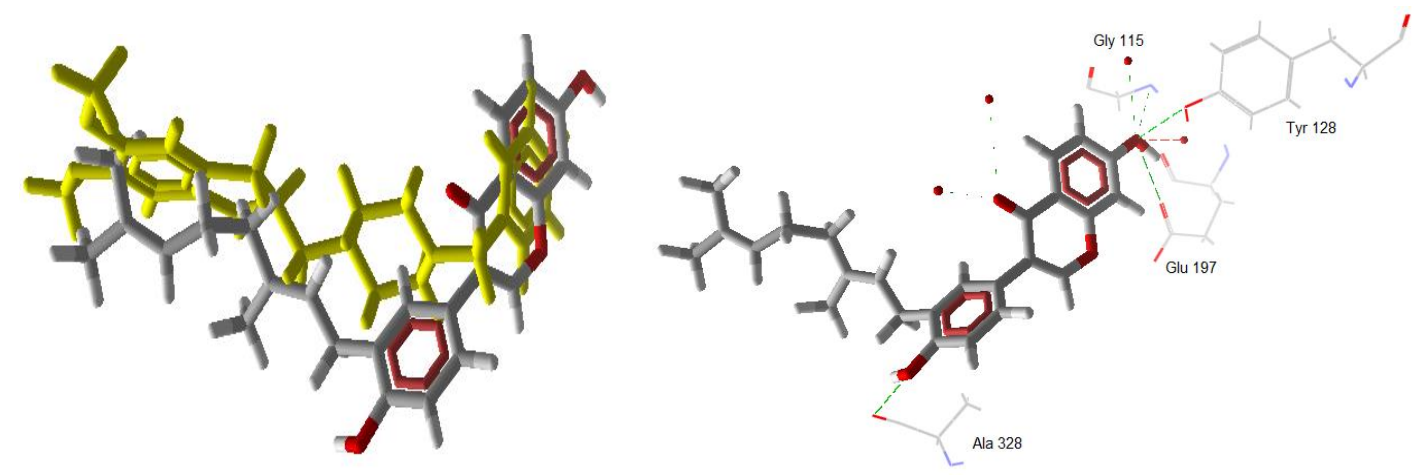


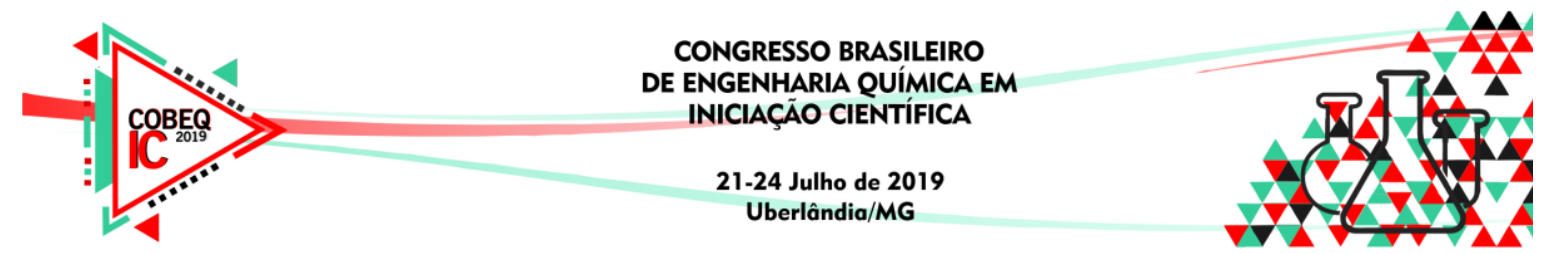

Figura 5 - Pose escolhida para o do Corylifol A na HssBChE em sobreposição ao donepezil de amarelo (à esquerda) e interações com os resíduos (à direita).
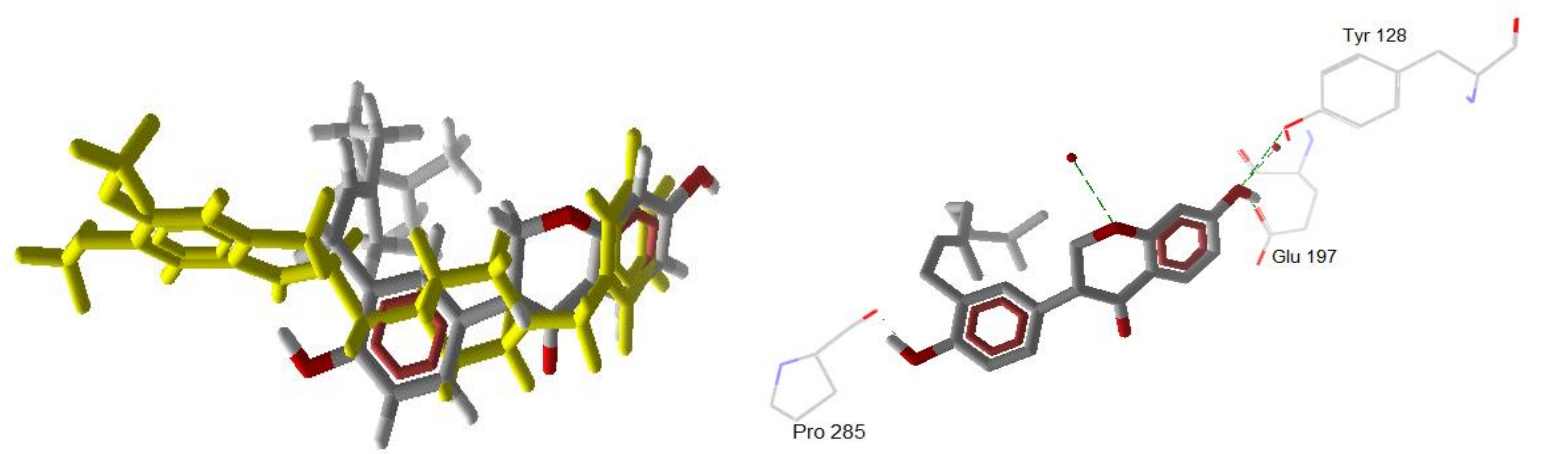

As melhores poses dos compostos com as melhores energias de interação em sobreposição ao donepezil, e as interações dos compostos com os resíduos pertencentes ao sítio ativo da $H s s \mathrm{BChE}$ mostradas acima é uma forma de obter uma melhor compreensão das diferenças entre os modos de interação de cada composto e os fatores responsáveis pela melhor afinidade com a enzima estudada.

\section{CONCLUSÃO}

Os estudos por docking sugerem o corylifol A e o Neobavaisoflavone como potenciais inibidores da Hss AChE e da $H s s \mathrm{BChE}$, além de identificar resíduos fundamentais para a interação inibidor-enzima para o planejamento de novos fármacos. Posteriormente, serão feitos estudos por dinâmica molecular desses compostos no sítio ativo das enzimas objetivando contribuir de forma significativa para o planejamento de novos protótipos mais eficazes e seletivos e consequentemente possíveis novos fármacos contra DA.

\section{REFERÊNCIAS}

1. CHEN, Z, J; Yang, Y, F; Zhang, Y, T; Yang, D, H. Molecules 2018, 23, 196.

2. FERRO, E. D. S; GUIMARÃES, A. P; GONÇALVES; A. S. L. Estudo de ancoramento molecular (docking) de potenciais inibidores da acetilcolinesterase humana (HssAChE). XXXII Encontro Regional da Sociedade Brasileira de Química. 2018.

3. HEHRE, W. J.; DEPPMEIER, B. J.; KLUNZINGER, P. E. PC SPARTAN Pro, Wavefunction Inc.: Irvine, Califórnia, 1999.

4. KONTOYIANNI, M.; MCCLELLAN, L. M.; SOKOL, G. S. Evaluation of Docking Performance: Comparative Data on Docking Algorithms. Journal of Medicinal Chemistry, v. 47, p. 558-565, 2004.

5. THOMSEN, R.; CHRISTENSEN, M. H. MolDock: A New Technique for High Accuracy Molecular Docking. Journal of Medicinal Chemistry, v. 49, p. 3315- 3321, 2006. 